\title{
Principais riscos e a importância do tratamento relacionados a gestação ectópica
}

\author{
Main risks and importance of treatment related to ectopic pregnancy
}

Principales riesgos e importancia del tratamiento relacionado con el embarazo ectópico

Iara Nadine Vieira da Paz Silva ORCID: https://orcid.org/0000-0002-5027-200X Faculdade Estácio de Sá, Brasil

E-mail: iaranadine15@ hotmail.com

Edmar José Fortes Júnior ORCID: https://orcid.org/0000-0002-0114-9937 Instituto de Ensino Superior do Vale do Parnaíba, Brasil

E-mail: edmarfortes@hotmail.com

Marcela Maria Lopes Rocha

ORCID: https://orcid.org/0000-0002-0189-3838 Instituto de Ensino Superior do Vale do Parnaíba, Brasil

E-mail: mamalrocha@outlook.com

Luiza Botelho Veloso

ORCID: https://orcid.org/0000-0002-0676-6168

Centro Universitário ITPAC Porto, Brasil

E-mail: lubotelhoveloso@ hotmail.com

José Guilherme Férrer Pompeu

ORCID: https://orcid.org/0000-0001-9038-0833

Universidade Federal do Piauí, Brasil

E-mail: gpompeu8@gmail.com

Eudes Rufino da Silveira Filho

ORCID: https://orcid.org/0000-0003-0360-1322

Centro Universitário Uninovafapi, Brasil

E-mail: personalgigaeudes@gmail.com

Melka Luciana Rocha de Paiva

ORCID: https://orcid.org/0000-0002-5483-0873

Universidade Federal do Piauí, Brasil

E-mail: Melkaluciana@gmail.com

Angelica Ribeiro do Nascimento Oliveira

ORCID: https://orcid.org/0000-0002-7240-2004

Centro Universitário Maurício de Nassau, Brasil

E-mail: angelicalribeiro.ar19@gmail.com

Flávia Lorena Henrique Dos Anjos

ORCID: https://orcid.org/0000-0002-7357-7754

Universidade Federal do Piauí, Brasil

E-mail: flavinha.lorenna@gmail.com

Daniely Lemos de Sousa

ORCID: https://orcid.org/0000-0002-0536-399X

Faculdade Estácio de Sá, Brasil

E-mail: daniely.lemos6@hotmail.com

Alessandro Monteiro Carvalho

ORCID: https://orcid.org/0000-0001-8257-6916

Universidade Federal do Piauí, Brasil

E-mail: alefarma443@yahoo.com.br

José Jonas Soares Guedes

ORCID: https://orcid.org/0000-0002-0858-3525

Centro Universitário Santo Agostinho, Brasil

E-mail: jonassguedes@hotmail.com

Milena Matos da Cruz

ORCID: https://orcid.org/0000-0002-9875-8906

Centro Universitário ITPAC Porto, Brasil

E-mail: milenamc_5@hotmail.com

Thais Rocha Silva

ORCID: https://orcid.org/0000-0002-7591-294X

Universidade Estadual do Maranhão, Brasil

E-mail: thaisrocha1993@hotmail.com

Mayara Rafaela dos Reis

ORCID: https://orcid.org/0000-0002-6973-004X

Centro Universitário Facid, Brasil

E-mail: mayara12reis@gmai.com 


\author{
Ana Lourdes dos Reis Silva \\ ORCID: https://orcid.org/0000-0002-8513-5953 \\ Faculdade Estácio de Sá, Brasil \\ E-mail: annalourdes33@Outlook.com \\ Maria Eliane Martins Oliveira da Rocha \\ ORCID: https://orcid.org/0000-0002-7743-6107 \\ Universidade Estadual do Piauí, Brasil \\ E-mail: memor10@hotmail.com
}

\begin{abstract}
Resumo
O presente artigo tem como objetivo descrever os principais riscos e a importância do tratamento relacionados a gestação ectópica. Trata-se de uma revisão Integrativa da Literatura, um estudo de síntese do conhecimento a partir da análise das evidências disponíveis, de modo sistemático e rigoroso. A questão norteadora desse estudo foi: Quais são os principais riscos e a importância do tratamento relacionados a gestação ectópica? Foram incluídos na pesquisa: artigos completos disponíveis de forma livre a gratuita, idiomas em português, inglês e espanhol. Excluindo-se artigos duplicados, indisponíveis e incompletos, Com recorte temporal de 2014 a 2021. O resgate dos estudos ocorreu a partir dos descritores controlados DeCS (Descritores em Ciências da Saúde): "gestação ectópica", "tratamento", "riscos" e "cuidados". As bases de dados consultadas foram: MEDLINE (Medical Literature Analysis and Retrieval System Online), LILACS (Literatura Latino-Americana do Caribe em Ciências da Saúde), Google acadêmico e a biblioteca virtual SciELO. A partir da coleta de dados, foram encontrados 705 estudos. A primeira fase da análise consistiu na aplicação dos critérios de inclusão e exclusão. Com isso conseguiu-se 7 artigo ao final da primeira fase. $\mathrm{Na}$ segunda fase, decorreu-se a leitura dos 6 estudos para encontrar aqueles que respondiam corretamente a pergunta norteadora da pesquisa e/ou tinham adequação com o fenômeno do estudo. Portanto mostra-se a relevância do tema abordado, bem como todas suas formas e apresentações que envolve desde a infertilidade até o óbito de muitas mulheres, e os estudos seguem mostrando a importância do diagnóstico precoce, bem como de tratamentos menos invasivos visando um menor impacto na vida das mulheres.
\end{abstract}

Palavras-chave: Gestação ectópica; Tratamento; Riscos; Cuidados.

\begin{abstract}
This article aims to describe the main risks and the importance of treatment related to ectopic pregnancy. This is an integrative literature review, a study of knowledge synthesis based on the analysis of available evidence, in a systematic and rigorous way. The guiding question of this study was: What are the main risks and importance of treatment related to ectopic pregnancy? The research included: complete articles available free of charge, languages in Portuguese, English and Spanish. Excluding duplicate, unavailable and incomplete articles, with a time frame from 2014 to 2021. The rescue of the studies occurred from the controlled descriptors DeCS (Descriptors in Health Sciences): "ectopic pregnancy", "treatment", "risks" and "care". The databases consulted were: MEDLINE (Medical Literature Analysis and Retrieval System Online), LILACS (Latin American Caribbean Literature in Health Sciences), academic Google and the SciELO virtual library. From the data collection, 705 studies were found. The first phase of the analysis consisted of applying the inclusion and exclusion criteria. With that, 7 articles were obtained at the end of the first phase. In the second phase, the reading of the 6 studies was carried out to find those that correctly answered the research's guiding question and/or were adequate to the phenomenon of the study. Therefore, the relevance of the topic discussed is shown, as well as all its forms and presentations that involve from infertility to the death of many women, and studies continue showing the importance of early diagnosis, as well as less invasive treatments aiming at a lower impact in women's lives.
\end{abstract}

Keywords: Ectopic pregnancy; Treatment; Risks; Care.

\title{
Resumen
}

Este artículo tiene como objetivo describir los principales riesgos y la importancia del tratamiento relacionado con el embarazo ectópico. Se trata de una revisión integradora de la literatura, un estudio de síntesis de conocimientos basado en el análisis de la evidencia disponible, de forma sistemática y rigurosa. La pregunta orientadora de este estudio fue: ¿Cuáles son los principales riesgos y la importancia del tratamiento relacionado con el embarazo ectópico? La investigación incluyó: artículos completos disponibles de forma gratuita, idiomas en portugués, inglés y español. Excluyendo artículos duplicados, no disponibles e incompletos, con un marco temporal de 2014 a 2021 . El rescate de los estudios se realizó a partir de los descriptores controlados DeCS (Descriptors in Health Sciences): "embarazo ectópico", "tratamiento", "riesgos" y "cuidado". Las bases de datos consultadas fueron: MEDLINE (Sistema de Análisis y Recuperación de Literatura Médica en Línea), LILACS (Literatura del Caribe Latinoamericano en Ciencias de la Salud), Google académico y la biblioteca virtual SciELO. De la recopilación de datos, se encontraron 705 estudios. La primera fase del análisis consistió en aplicar los criterios de inclusión y exclusión. Con eso, se obtuvieron 6 artículos al final de la primera fase. En la segunda fase, se realizó la lectura de los 7 estudios para encontrar aquellos que respondieran correctamente a la pregunta orientadora de la investigación y / o fueran adecuados al fenómeno del estudio. Por tanto, se muestra la relevancia del tema tratado, así como todas sus formas y presentaciones que involucran desde la infertilidad hasta la muerte de muchas mujeres, y los estudios continúan mostrando la importancia del diagnóstico precoz, así como 
de tratamientos menos invasivos orientados a un menor impacto en la vida de las mujeres.

Palabras clave: Embarazo ectópico; Tratamiento; Riesgos; Cuidado.

\section{Introdução}

Durante o estado gravídico, a mulher estar sujeita a mudanças no processo metabólico. De modo geral, os fatores de riscos que podem tornar o prognóstico materno e fetal desfavorável são os aspectos individuais, situação sociodemográficas desfavorável, histórico reprodutivo condições clínicas e obstétrica isolada ou associada a outros problemas que repercutem na evolução da gestação (Barbosa et al., 2020). A gravidez ectópica é uma complicação da gestação, na qual o feto se aloja fora do útero, ela retrata cerca de $1 \%$ das gestações. Entre os locais onde ela começa a se desenvolver, o mais comum seria na trompa uterina, que ocorre em cerca de $95 \%$ dos casos, nos casos atípicos localiza-se no ovário, colo uterino, cavidade abdominal e cicatriz de cesárea (Nascimento et al., 2019).

Com isso, os sintomas se manifestam de acordo com o local onde a gestação está localizada, contudo os mais comumente são atrasos menstruais, sangramento genital e dor abdominal, que em geral está relacionado com a gravidez na região da trompa. E os fatores de risco relacionados seriam tabagismo, infeções sexualmente transmissíveis, como doença inflamatória pélvica e infecção por Chlamydia trachomatis; abortos espontâneos prévios; idade superior a 40 anos; técnicas de reprodução assistida; número de parceiros sexuais; uso de dispositivo intrauterino (DIU), podendo estar associado ainda com cirurgia ginecológica, infertilidade, histórico de placenta prévia, fertilização in vitro, anomalias congênitas do útero (Nascimento et al., 2019).

Se estas complicação não for diagnosticada no início, antes de oito semanas de gravidez, a vida da mãe corre sérios riscos, pois a evolução dessa gestação a placenta pode se introduzir nos tecidos e órgãos vizinhos, além de romper vasos calibrosos e causar hemorragias intensas, tendo que ser feita histerectomia de urgência e podendo levar ao óbito materno. Portanto, a falta de informação das gestantes e inicialização tardia do pré-natal podem atrasar o diagnóstico e a devida assistência, podendo gerar sequelas ou morte da mãe e do filho. Sendo seu diagnóstico realizado através da dosagem seriada de $\beta$-HCG e da ultrassonografia transvaginal (Fernandes; Lima, 2018).

Portanto o tratamento pode ser clínico ou cirúrgico. O medicamento mais utilizado é o Metotrexato. O uso dessa medicação pode ser sistêmico ou local. A injeção local é aplicada quando o embrião está vivo, em situações que não sugeria o procedimento cirúrgico, sendo utilizada uma dose de $1 \mathrm{mg} / \mathrm{kg}$ guiada por ultrassonografia transvaginal e sedação da paciente. Já o metotrexato sistêmico tem maior êxito no tratamento clínico em gestação menor que 6 semanas, com massa tubária menor que 3,5 $\mathrm{cm}$ de diâmetro, feto morto e $\beta-\mathrm{HCG}$ inferior a $1500 \mathrm{mUI} / \mathrm{ml}$. Pacientes hemodinamicamente instáveis, com sinais de ruptura, $\beta$ HCG superior a $5000 \mathrm{mUI} / \mathrm{ml}$, necessidade de diagnóstico laparoscópico ou suspeita de gestação heterotópica devem ser submetidas a intervenção cirúrgica. Também contribuem para essa indicação, massa anexial maior que $4 \mathrm{~cm}$ de diâmetro e líquido livre na pelve visto na ultrassonografia transvaginal (Klaesener, 2018).

Além disso, o valor inicial do $\beta$-HCG é um preditor para o conquista da terapia, sendo que quanto menor o valor maior o êxito terapêutico. Dentre esses valores quanto mais baixo melhor a resolução com uma dose, e quanto mais alto maior a necessidade de uma segunda dose, sendo que essa segunda dose possui uma taxa de fracasso com medidas de $\beta$-HCG maiores. Dessa forma, pacientes tratadas com metotrexato devem ser acompanhadas de perto e à medida que os valores de $\beta$-HCG forem verificados o médico deve deixa-la ciente do que ocorre no transcorrer do tratamento alertando a ela sobre a possibilidade de uma cirurgia caso os valores do $\beta$-HCG não reduzam como o desejado ou continue aumentando (Nether et al., 2019).

O presente artigo tem como objetivo descrever os principais riscos e a importância do tratamento relacionados a gestação ectópica. 


\section{Metodologia}

Trata-se de uma revisão Integrativa da Literatura, um estudo de síntese do conhecimento a partir da análise das evidências disponíveis, de modo sistemático e rigoroso. Desse modo, cumprindo o rigor metodológico para a elaboração de uma revisão integrativa, cumpriu-se as seis etapas: identificação do tema e seleção da questão de pesquisa; estabelecimento de critérios para inclusão e busca de estudos na literatura; definição das informações a serem extraídas dos estudos selecionados; avaliação dos estudos incluídos na revisão integrativa; interpretação dos resultados e apresentação da síntese do conhecimento (Ercole; Melo \& Alcoforado; 2014; Mendes; Silveira \& Galvão, 2019).

A questão norteadora desse estudo foi: Quais são os principais riscos e a importância do tratamento relacionados a gestação ectópica?

Foram incluídos na pesquisa: artigos completos disponíveis de forma livre a gratuita, idiomas em português, inglês e espanhol. Excluindo-se artigos duplicados, indisponíveis e incompletos, Com recorte temporal de 2014 a 2021.

O resgate dos estudos ocorreu a partir dos descritores controlados DeCS (Descritores em Ciências da Saúde): “gestação ectópica", "tratamento", "riscos" e "cuidados". As bases de dados consultadas foram: MEDLINE (Medical Literature Analysis and Retrieval System Online), LILACS (Literatura Latino-Americana do Caribe em Ciências da Saúde), Google acadêmico e a biblioteca virtual SciELO.

\section{Resultados e Discussão}

A partir da coleta de dados, foram encontrados 705 estudos. A primeira fase da análise consistiu na aplicação dos critérios de inclusão e exclusão. Com isso conseguiu-se 6 artigo ao final da primeira fase. Na segunda fase, decorreu-se a leitura dos 7 estudos para encontrar aqueles que respondiam corretamente a pergunta norteadora da pesquisa e/ou tinham adequação com o fenômeno do estudo.

O Quadro 1 apresenta uma visão geral dos artigos, em que se pode identificar os autores e ano, nome do artigo, objetivo e conclusão.

Quadro 1: Síntese dos artigos encontrados e inclusos na revisão.

\begin{tabular}{|c|c|c|c|}
\hline NOME DO ARTIGO & AUTOR/ANO & OBJETIVO & CONCLUSÃO \\
\hline $\begin{array}{c}\text { Tratamento Clínico De } \\
\text { Gravidez Ectópica Com Uso Do } \\
\text { Metotrexate }\end{array}$ & $\begin{array}{l}\text { (Peixoto; } \\
\text { Souza; } \\
\text { Miranda, } \\
\text { 2017). }\end{array}$ & $\begin{array}{l}\text { Analizar a importancia do } \\
\text { Tratamento Clínico De } \\
\text { Gravidez Ectópica Com Uso } \\
\text { Do Metotrexate. }\end{array}$ & $\begin{array}{l}\text { Se faz cada vez mais necessário o acompanhamento } \\
\text { pré-natal desde o início da gestação, afim de } \\
\text { identificar possíveis complicações e condições com a } \\
\text { maior antecedência possível, de forma que a conduta } \\
\text { escolhida, seja ela qual for, possa garantir a saúde e a } \\
\text { integridade da paciente. }\end{array}$ \\
\hline $\begin{array}{l}\text { A incidência de uma gravidez } \\
\text { ectópica e sua relação com o } \\
\text { quadro de infertilidade }\end{array}$ & $\begin{array}{c}\text { (Santos; } \\
\text { Souza, 2021). }\end{array}$ & $\begin{array}{l}\text { Realizar um levantamento } \\
\text { epidemiológico do número de } \\
\text { casos de Gravidez Ectópicas } \\
\text { (GE) confirmadas, na Rede } \\
\text { Pública de Saúde no Estado de } \\
\text { São Paulo. }\end{array}$ & $\begin{array}{c}\text { O seguinte trabalho expõe de forma sigilosa a } \\
\text { seguinte evidência, dos } 30 \text { dados epidemiológicos } \\
\text { estudados } 10 \text { mulheres apresentam quadros de } \\
\text { infertilidade em função do descobrimento tardio e } \\
\text { tratamento cirúrgico. Portanto, se faz necessário que o } \\
\text { diagnóstico aconteça antes do segundo trimestre } \\
\text { gestacional. }\end{array}$ \\
\hline $\begin{array}{c}\text { Avaliação do teste Inexscreen }{ }^{\circledR} \\
\text { para o diagnóstico de gravidez } \\
\text { ectópica e Abortamento }\end{array}$ & $\begin{array}{l}\text { (Teixeira, } \\
\text { 2019). }\end{array}$ & $\begin{array}{l}\text { Avaliar o desempenho } \\
\text { diagnóstico do Inexscreen para } \\
\text { o diagnóstico de gestações } \\
\text { anormais (gravidez ectópica e } \\
\text { abortamento). }\end{array}$ & $\begin{array}{c}\text { Como conclusão os resultados apresentados em nosso } \\
\text { trabalho (Em uma emergência), não foram possíveis } \\
\text { para validar se o teste comercial é adequado para } \\
\text { detectar ou excluir uma gravidez anormal no primeiro } \\
\text { trimestre. }\end{array}$ \\
\hline $\begin{array}{l}\text { Tratamento conservador da } \\
\text { gravidez ectópica. }\end{array}$ & $\begin{array}{l}\text { (Morais et al., } \\
\text { 2021). }\end{array}$ & $\begin{array}{c}\text { Discutir as opções de } \\
\text { tratamento não } \\
\text { invasivo/conservador no } \\
\text { contexto da gravidez ectópica. }\end{array}$ & $\begin{array}{l}\text { Conclui-se que o tratamento não invasivo se mostra } \\
\text { relevante por meio da segurança e eficácia do MTX e } \\
\text { pela alternativa da terapia expectante, determinados a } \\
\text { partir do valor preditivo do beta-HCG, em contraste } \\
\text { com os riscos inerentes ao tratamento invasivo. }\end{array}$ \\
\hline
\end{tabular}




\begin{tabular}{|c|c|c|c|}
\hline $\begin{array}{c}\text { Fatores De Risco Para Gravidez } \\
\text { Ectópica Em Um Hospital } \\
\text { Universitário }\end{array}$ & $\begin{array}{l}\text { (Quessada, } \\
\text { 2017). }\end{array}$ & $\begin{array}{l}\text { Avaliar a incidência, os fatores } \\
\text { de risco e a presença de sinais e } \\
\text { sintomas das mulheres no } \\
\text { primeiro trimestre gestacional, } \\
\text { atendidas no Setor de } \\
\text { Emergência Ginecológica do } \\
\text { Hospital de Clínicas de Porto } \\
\text { Alegre (HCPA). }\end{array}$ & $\begin{array}{l}\text { A incidência diagnóstica confirmada de GE foi de } \\
\text { 8,5\% (95\% IC= } 6.8 \text { a 10.6). Entre os fatores de risco } \\
\text { para GE, os que apresentaram maior risco relativo } \\
\text { foram, respectivamente, GE prévia e história de } \\
\text { cirurgia tubária. Os sinais e sintomas mais relevantes } \\
\text { para o diagnóstico de GE foram dor mais } \\
\text { sangramento, que estão fortemente, relacionadas ao } \\
\text { diagnóstico de GE. }\end{array}$ \\
\hline $\begin{array}{l}\text { Protocolo Brasileiro para } \\
\text { Infecções Sexualmente } \\
\text { Transmissíveis 2020: doença } \\
\text { inflamatória pélvica }\end{array}$ & $\begin{array}{l}\text { (Menezes et } \\
\text { al., 2021). }\end{array}$ & $\begin{array}{l}\text { Abordar a doença inflamatória } \\
\text { pélvica, tema que compõe o } \\
\text { Protocolo Clínico e Diretrizes } \\
\text { Terapêuticas (PCDT) para } \\
\text { Atenção Integral às Pessoas } \\
\text { com Infecções Sexualmente } \\
\text { Transmissíveis }\end{array}$ & $\begin{array}{l}\text { Gestantes com doença inflamatória pélvica têm alto } \\
\text { risco de abortamento, corioamnionite e parto } \\
\text { prematuro, devendo ser internadas e iniciar } \\
\text { imediatamente antibióticos intravenosos de amplo } \\
\text { espectro. Doxiciclina e quinolonas são } \\
\text { contraindicadas na gestação. }\end{array}$ \\
\hline $\begin{array}{l}\text { Tratamento clínico da prenhez } \\
\text { ectópica }\end{array}$ & $\begin{array}{l}\text { (Magalhães; } \\
\text { Jesus; Trajano, } \\
\text { 2015). }\end{array}$ & $\begin{array}{c}\text { Revisar o manejo } \\
\text { medicamentoso e conservador } \\
\text { da gestação ectópica, visando } \\
\text { estabelecer a melhor seleção e } \\
\text { abordagem nesta situação }\end{array}$ & $\begin{array}{l}\text { A mudança do perfil de apresentação clínica das } \\
\text { pacientes com gestação ectópica abriu espaço para a } \\
\text { adoção de condutas mais conservadoras, visando } \\
\text { preservar o futuro reprodutivo. O tratamento } \\
\text { medicamentoso com metotrexato é consagrado na } \\
\text { literatura, apresentando boa resposta clínica e taxas de } \\
\text { gestação tópica subsequente consideráveis. }\end{array}$ \\
\hline
\end{tabular}

Fonte: Autores.

As informações a serem discutidas foram categorizadas para uma melhor análise, interpretação e esclarecimento das temáticas abordadas. Optou-se por dividir em 3 tópicos (categorias), buscando uma melhor associação entre os resultados encontrados na literatura.

\subsection{Gestação ectópica}

Algumas gestações ectópicas efetuam o aborto espontaneamente em contrapartida outras continuam a se desenvolver causando rupturas, levando a gestante a correr sérios ricos. Sendo considerada como a principal causa de morte materna no primeiro trimestre da gestação. Nos últimos anos a literatura médica tem publicado diversos relatos de casos de gestação ectópica de localização atípica (intersticial, cervical, cicatriz de cesárea, ovariana e abdominal), os ricos à gestantes não se isentam pois a implantação do óvulo em lugares inapropriados pode causar grave lesão das estruturas que rodeiam que representam situações de elevada morbimortalidade, como representado na Figura 1 (Peixoto; Souza; Miranda, 2017). 
Figura 1: Implantação normal do embrião no útero (gravidez normal) e falha na implantação do embrião na trompa de Falópio.

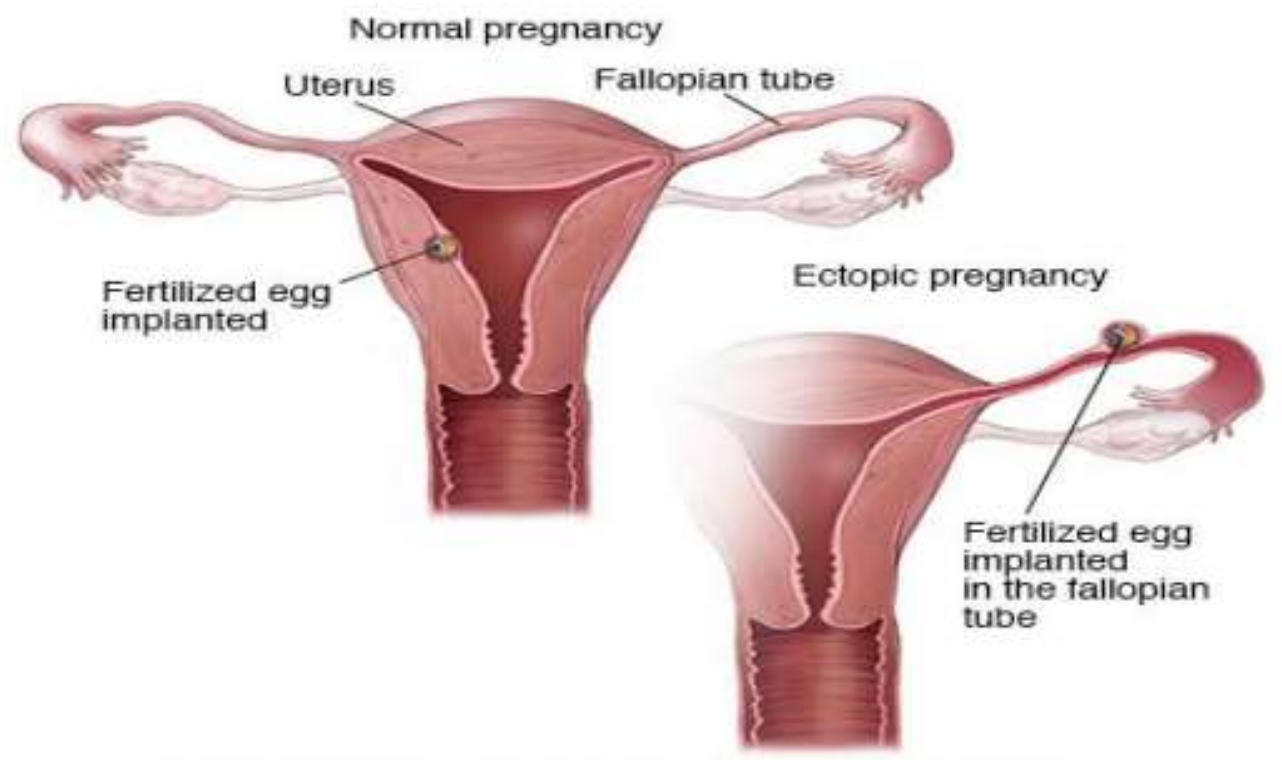

Fonte: Adaptado de (Teixeira, 2019).

Figura 2: Locais onde ocorrem falhas na implantação do embrião.

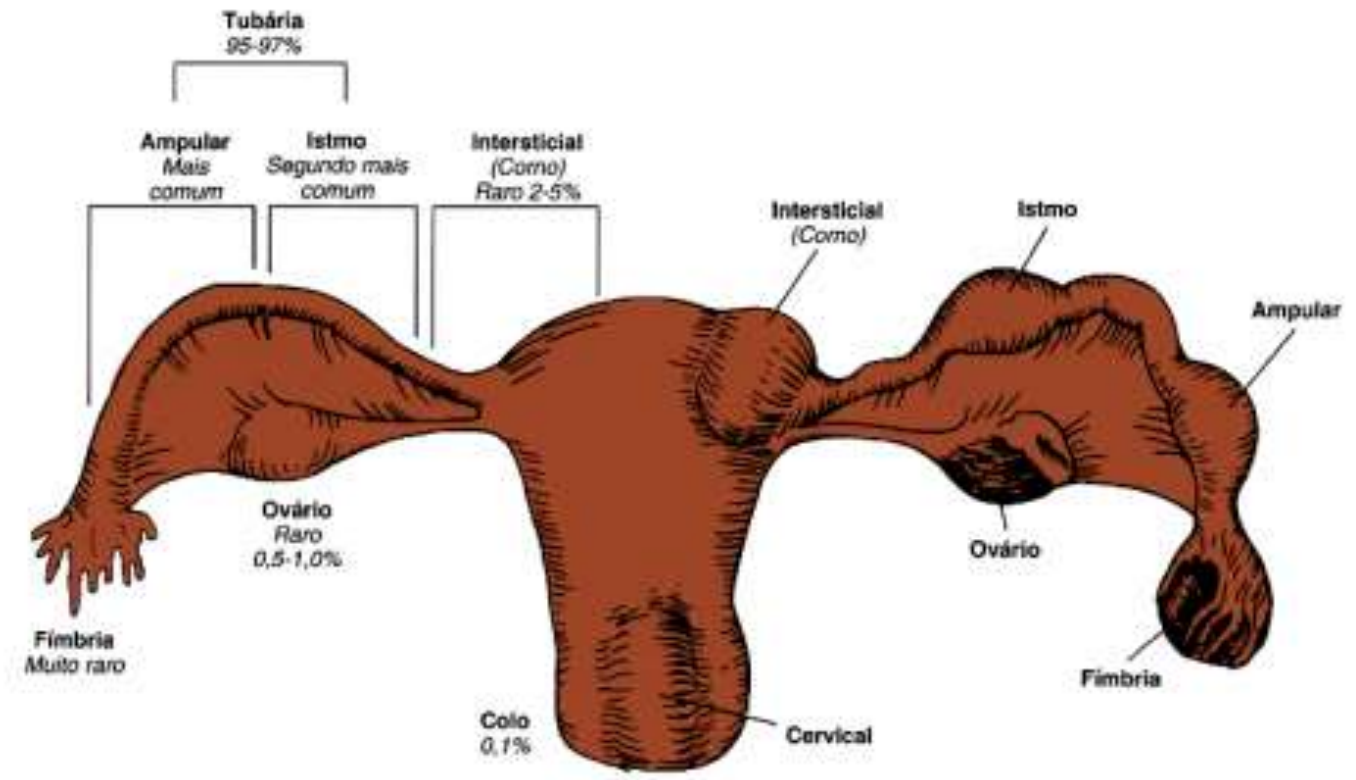

Fonte: Adaptado de (Teixeira, 2019).

Além disso, a gestação ectópica é mais comum em mulheres que fazem tratamento para infertilidade, inclusive na inexistência de doença tubária. A tecnologia de reprodução assistida por fecundação in vitro aumenta a taxa de gestação ectópica em aproximadamente 2 a 5\%. O motivo para o aumento dessa incidência ainda não foi atestada, mas acredita-se que esteja relacionada com a técnica de transferência de embriões. Entende-se que a gestação ectópica tanto pode estar relacionado à infertilidade como também pode causá-la, fenômeno esse que pode tornar essa associação muito complexa. Dessa forma, o diagnóstico precoce é importante, pois o diagnóstico tardio apresenta um risco imediato à saúde para a mulher, sendo capaz de 
levar a infertilidade, devido à possibilidade de lesão nas trompas que causa dificuldade na passagem do óvulo fertilizado (Quessada, 2017).

No que se refere à evolução clínica da gravidez ectópica, os sintomas mais descritos são dor e sangramento vaginal - que é de pequena monta e ocorre após atraso menstrual. Nas situações de maior gravidade pode ocorrer rotura da gravidez ectópica, com acentuação do hemoperitônio e generalização da dor para todo o abdome. Sendo assim, ao exame físico percebem-se sinais de choque hipovolêmico e intensa dor ao toque - grito de Douglas ou Sinal de Proust. Por ser uma condição potencialmente grave e em muitos casos um desafio, é importante realizar o diagnóstico precoce da gravidez ectópica, preferencialmente antes da rotura tubária (Morais et al., 2021).

\subsection{Principais riscos da gestação ectópica}

Alguns trabalhos analisados, foram encontrados vários relatos que relacionam a idade a uma maior evidência de casos de gestação ectópica. Entre as várias explicações para a vinculação entre idade materna e gestação ectópica, a mais importante delas é que mulheres com idade mais avançada foram por muito mais tempo expostas aos fatores de risco, o que provocam diversas mudanças na motilidade tubária, resultante de atraso do transporte do ovo e posterior implantação deste na tuba. Há também a hipótese que alterações hormonais do próprio corpo, com o passar dos anos, venham a provocar as mesmas alterações já citadas. Com relação ao fator paridade, a condição mais aceita é que as diversas gestações podem causar alterações anatômicas e funcionais ao próprio processo de gestação intrauterina, o que pode levar as modificações de motilidade e implantação (Peixoto; Souza; Miranda, 2017).

Contudo o tabagismo é uma das causas mais relevantes para o risco de gestação ectópica em mulheres na fase adulta, porem os números em adolescentes mostram-se relevantes também. Dados epidemiológicos constatam que frequentemente mulheres que fumam têm riscos com maior variedade de problemas reprodutivos, a exposição a fumaça (fumante primário ou secundário), intensifica o risco de aborto espontâneo, restrição do crescimento intrauterino, ruptura prematura de membranas, parto prematuro, hematoma retroplacentário, placenta prévia, e da morte súbita do lactente (Teixeira, 2019).

\subsection{Formas de tratamento relacionados a gestação ectópica}

Para ser iniciado o tratamento antes deve ser realizado exames clínicos e laboratoriais. O exame clínico deve obter verificação de sinais vitais; exame abdominal; exame especular vaginal, com inspeção do colo de útero para friabilidade (sangramento fácil) e corrimento mucopurulento cervical; toque vaginal bimanual, com mobilização do colo; e palpação dos anexos (ovários e trompas uterinas). E os seguintes exames de laboratório: hemograma completo, enzimas hepáticas (TGO e TGP), creatinina e tipagem sanguínea AB0-Rh. Deve ser feita também dosagem seriada de beta-HCG com intervalo de 48 horas previamente ao início do tratamento objetivando confirmar a ascensão dos títulos (Menezes et al., 2021).

O medicamento utilizado no tratamento medicamentoso é o metotrexato e deve ser concedido como primeira linha no tratamento, o beta-HCG menor que $1.500 \mathrm{UI} / \mathrm{l}$ e naquelas pacientes com valores de beta-HCG entre 1.500 e $5.000 \mathrm{UI} / \mathrm{ml}$, o tratamento medicamentoso também pode ser apresentado como opção ao tratamento cirúrgico, pois, apesar destes valores apresentem taxas de falha e necessidade de intervenção maiores, as taxas de sucesso ainda são significativas. As contraindicações absolutas são: gravidez intrauterina; imunodeficiência; anemia moderada a intensa, leucopenia ou trombocitopenia; sensibilidade prévia ao metotrexato, na vigência de doença pulmonar e úlcera péptica; disfunção importante hepática e renal; amamentação. As contraindicações relativas são batimentos cardíacos fetais presentes, beta-HCG maior que $5.000 \mathrm{UI} / \mathrm{ml}$ e pacientes que se recusa em receber transfusão sanguínea (Magalhães; Jesus; Trajano, 2015).

Existem dois esquemas sancionado para administração do metotrexato o de dose única e o de múltiplas doses. No 
esquema de dose única, é administrado $50 \mathrm{mg} / \mathrm{m}^{2}$ de metotrexato via intramuscular. A supervisão se faz através de dosagens do beta-HCG, realizadas no mesmo dia e no sétimo dia após o emprego do medicamento, as taxas do hormônio devem ser acompanhados até ficarem negativos, caso não ocorra queda das taxas, pode ser administrada outra dose do medicamento. Em média, os títulos de beta-HCG negativam em três semanas. Entretanto, pacientes com valores mais altos podem levar seis a oito semanas para atingirem este objetivo. O esquema de múltiplas doses seria na administração intramuscular de $1 \mathrm{mg} / \mathrm{kg}$ de metotrexato nos dias um, três, cinco e sete, intermitente com a utilização de ácido folínico na dose de $0,1 \mathrm{mg} / \mathrm{kg}$ nos dias dois, quatro, seis e oito. O seguimento é feito com a dosagem de beta-HCG no dia da dose inicial e antes de cada nova dose. O acompanhamento nos casos com boa resposta é semelhante ao de dose única, com dosagens de beta-HCG semanais até a negativação (Magalhães; Jesus; Trajano, 2015).

Outras alternativas para o tratamento, pode-se incluir a Fertilização In Vitro e a Inseminação Artificial, ambos são técnicas que utilizam medicamentos para induzir a ovulação para realizar a fecundação. A Inseminação Artificial é um procedimento de menor complexidade em comparação com a fertilização in vitro, onde consiste em diminuir o caminho percorrido pelo espermatozoide até o óvulo, o sêmen é colocado diretamente na cavidade uterina, no período de ovulação da mulher, para ser fecundado na tuba uterina. Nos casos em que há modificações nas trompas ou que há deformações importantes do sêmen. A Fertilização In Vitro é um procedimento realizado em laboratório, sendo realizada uma punção transvaginal e retirado o óvulo do ovário, em seguida, é fecundado pelo espermatozoide no laboratório, fora do corpo feminino. Após alguns dias de desenvolvimento, o embrião que se encontrava no laboratório é transferido para o útero (Santos; Souza, 2021).

\section{Considerações Finais}

Portanto mostra-se a relevância do tema abordado, bem como todas suas formas e apresentações que envolve desde a infertilidade até o óbito de muitas mulheres, e os estudos seguem mostrando a importância do diagnóstico precoce, bem como de tratamentos menos invasivos visando um menor impacto na vida das mulheres.

\section{Referências}

Barbosa, M. L., Barbosa, A. L., Barbosa, T. P., Silva, R. C. C. D., Almeida, P. C. D., \& Libório, A. B. (2020). Perfil clínico-epidemiológico de mulheres no ciclo gravídico-puerperal internadas em uma Unidade de Terapia Intensiva.

Ercole, F. F., Melo, L.S., \&Alcoforado, C. L. G. C. Revisão integrativa versus revisão sistemática (2014). Rev. REME, 18(1), 1-2.

Fernandes, K. V. M. L., \& Lima, C. B. (2018) Gravidez Ectópica: Reflexões Acerca Da Assistência De Enfermagem Ectopic Pregnancy: Reflections On Nursing Care.

Klaesener, C. (2018). Perfil clínico e epidemiológico de pacientes atendidas na emergência de ginecologia de um hospital terciário.

Magalhães, A. L., de Jesus, N. R., \& Trajano, A. J. (2015). Tratamento clínico da prenhez ectópica. Revista Hospital Universitário Pedro Ernesto (TíTULO NÃOCORRENTE), 14(2).

Mendes, K. D. S.,Silveira, R. C. C. P., \& Galvão, C. M.(2018). Uso de gerenciador de referências bibliográficas na seleção dos estudos primários em revisão integrativa. Texto contexto enferm, 28, e20170204.

Menezes, M. L. B., Giraldo, P. C., Linhares, I. M., Boldrini, N. A. T., \& Aragón, M. G. (2021). Protocolo Brasileiro para Infecções Sexualmente Transmissíveis 2020: doença inflamatória pélvica. Epidemiologia e Serviços de Saúde, 30 , e2020602.

Morais, L. R., Barreira, B. S. M., Saldanha, D. C., de Souza, F. P. G., Machado, L. C., de Paula Gomes, M., \& de Lima, M. M. B. (2021). Tratamento conservador da gravidez ectópica. Brazilian Journal of Health Review, 4(3), 13250-13260.

Nascimento, J. L. B., Zanovello, S. C., Galbarini, T. M. D. C. F., \& de Oliveira, D. M. C. (2019). Cuidados de enfermagem frente aos riscos evidenciados na gravidez ectópica. Brazilian Journal of Health Review, 2(2), 1444-1454.

Nether, G. M., Porto, M. S. R., Guerra, N. G., de Oliveira, B. F., \& Pedreira, J. H. G. (2019). Tratamento Conservador da Gravidez Ectópica. Cadernos da Medicina-UNIFESO, 2(2).

Peixoto, R. L., Souza, R. M., \& Miranda, F. (2017). Tratamento clínico de gravidez ectópica com uso do metotrexate. Revista rede de cuidados em saúde, 11(2). 
Research, Society and Development, v. 10, n. 9, e15410917810, 2021

(CC BY 4.0) | ISSN 2525-3409 | DOI: http://dx.doi.org/10.33448/rsd-v10i9.17810

Santos, V. S. V., \& de Souza, G. S. (2021). A incidência de uma gravidez ectópica e sua relação com o quadro de infertilidade. Brazilian Journal of Health Review, 4(3), 9669-9676.

Quessada, M. A. (2017). Fatores de risco para gravidez ectópica em um hospital universitário.

Teixeira, J. L. G. (2019). Avaliação do teste Inexscreen® para o diagnóstico de gravidez ectópica e abortamento. 